\title{
Phenylalanine Levels of Patients with Classical Phenylketonuria According to Eating Habits of Caregivers
}

\author{
(D) Pelin Teke Kısa1, (D) Abdurrahman Çiçek², (D) Hüseyin Karagöz², (D) Mahmut Dağ², (D) Arda Güneş², (D) Gülşah Yavaş², \\ (D) Nur Arslan1
}

${ }^{1}$ Dokuz Eylül University Faculty of Medicine, Department of Pediatric Metabolism and Nutrition, İzmir, Turkey

2Dokuz Eylül University Faculty of Medicine, İzmir, Turkey Cite this article as: Teke Kısa P, Çiçek A, Karagöz H, Dağ M, Güneş A, Yavaş G, Arslan N. Phenylalanine levels of classical phenylketonuria patients according to
eating habits of caregivers. Trends in Pediatrics 2021;2(4):154-158

\begin{abstract}
Objective: Diet is the main component in the treatment of classical phenylketonuria (PKU). Living in the same house as a PKU patient affects the lifestyle of family members in many ways. The present study examines the dietary habits of the parents of PKU patients.

Methods: The parents of 32 PKU patients were asked about their socio-demographic characteristics, family dietary patterns (mealtimes; frequency of meals with the PKU patient; content of foods at mealtimes, etc.) the parental dietary regimen and any food allergies.

Results: The mean age of the PKU patients was $11.5 \pm 4.3$ years, and 14 (44\%) were female. The mean plasma phenylalanine (Phe) level over the previous year was $732.4 \pm 339.0 \mu \mathrm{mol} / \mathrm{L}$ in the patient group. Within the study sample, $2(6 \%)$ families prepared only low-protein meals in the home, and the Phe levels of the two PKU patients in these families never exceeded $>600 \mu \mathrm{mol} / \mathrm{L}$, and $26(81 \%)$ parents prepared separate meals for the PKU patient and for the other family members every day. The $28(88 \%)$ parents had no special dietary regimen, with a mean Phe level of $774.8 \pm 345.4 \mu \mathrm{mol} / \mathrm{L}(\mathrm{p}=0.055)$. Finally, $26(81 \%)$ of the parents were careful about the foods they consumed while eating with the PKU patient, opting especially for foods that are low in Phe.

Conclusion: Diet is an indispensable part of the treatment of PKU and can affect the dietary patterns also of parents. Phe levels are lower in the children of parents who adopt a protein-restricted diet as a lifestyle, such as vegan and pescetarian. Large-scale studies are needed to investigate the physical, social and psychological effects of parents' dietary habits on PKU patients.
\end{abstract}

Keywords: Phenylketonuria, mealtime, parents, phenylalanine, diet, family

\section{INTRODUCTION}

Phenylketonuria (PKU) is the most common inborn error of the amino acid metabolism globally. In PKU, phenylalanine (Phe), an essential amino acid, cannot be converted into tyrosine due to a deficiency of phenylalanine hydroxylase enzyme activity in the liver. The most severe form of this disorder is known as classical PKU. Classical PKU occurs when phenylalanine hydroxylase activity is severely reduced or absent. Phe accumulates and the production of phenylketones is increased, resulting in high Phe levels in the blood and brain. Consequently, high blood levels of Phe have neurotoxic effects, and can lead to such serious neurological and psychiatric symptoms as microcephaly, mental retardation, epilepsy, behavioral disorder, hyperactivity and autism. ${ }^{1}$ Although the exact pathogenesis of neurocognitive dysfunction yet to be clearly explained, ${ }^{2}$ the goal with all available treatments is to reduce blood Phe levels, since it is known that high blood levels of Phe are strongly associated with neurocognitive dysfunction. The

P. Teke Kısa: 0000-0001-6481-3172; A. Çiçek: 0000-0003-0392-1932; H. Karagöz: 0000-0001-6012-2024; M. Dağ: 0000-0001-7581-6918; A. Güneş: 0000-0001-8594-3152; G. Yavaş: 0000-0001-6928-533X 
target is to maintain a blood Phe level of $<360 \mu \mathrm{mol} / \mathrm{L}$ in children under 12 years of age, while the target blood level of Phe is $<600$ $\mu \mathrm{mol} / \mathrm{L}$ in older children. ${ }^{1}$

The mainstay treatment is a lifelong Phe-restricted diet, ${ }^{3}$ which has three main features: (a) natural protein is restricted, (b) Phefree-L-amino acid supplements are used to provide the necessary protein, and (c) low-protein foods are consumed. ${ }^{1}$ The diet lacks high protein foods such as meat, chicken, fish, eggs, cheese and milk, with the calories that the patients need being provided by foods that are rich in carbohydrates and low in protein, such as fruit, vegetables and grains. ${ }^{3}$ Especially, children with classical PKU usually only tolerate Phe levels between $200-500 \mathrm{mg}$ day. ${ }^{1}$

The importance of family meals has been well established, however dining with a dieter can lead to changes in the eating habits and also in food preferences of other family members. To date, there has been a lack of studies investigating the eating habits of families of patients with classical PKU. The present study analyzes the eating habits of family members with a child with classical PKU so as to identify the strategies followed by families to cope with the associated problems.

\section{MATERIALS and METHODS}

This prospective study was conducted at Dokuz Eylül University Faculty of Medicine, a specialized PKU clinic. All the patients were diagnosed as having classical PKU (Phe $>1200 \mu \mathrm{mol} / \mathrm{L}$, Phe tolerance $<20 \mathrm{mg} / \mathrm{kg} /$ day) via Newborn Screening Program and their treatments were initiated immediately after their diagnosis. Phe levels were monitored based on recommendations of European guidelines on PKU (Frequency of blood Phe levels fortnightly at 1-12 years; monthly after the age of 12 years). ${ }^{1}$ Among the 37 eligible families, five caregivers declined to participate in the study, and so a total of 32 caregivers of children with classical PKU were enrolled in the study, who were asked to complete four questionnaires, and thus to provide details of:

1) The socio-demographic characteristics of the child and the caregivers; child's age, gender and dietary compliance; parent's age, sex, marital status, educational level, occupation,

2) Family dietary patterns (mealtimes; frequency of meals with the patient with PKU; content of foods at mealtimes, number of variety, frequency of foods consumed, etc.),

3) The parental dietary regimen (whether a pescatarian, vegetarian or vegan dietary regimen was followed),

4) Any food allergies (whether families were following a special diet).

\section{Statistical Analysis}

The data analysis was performed using IBM Statistical Package for the Social Sciences Statistics for Windows, Version 22.0 (SPSS IBM, Armonk, NY, USA). The normality of the sample distribution was tested using the Kolmogorov-Smirnov test. Numerical variables were expressed as mean \pm standard deviation or median (minimum- maximum), depending on the normality of distribution; and categorical variables were expressed as numbers and percentages (\%). Since the Phe levels were normally distributed; the Student's t-test and one-way ANOVA was used to compare the parameters between the groups. The level of statistical significance was set at a $p$-value of $<0.05$.

\section{Ethical Consideration}

The protocol of the present study was designed to be in compliance with the 1964 Declaration of Helsinki. The study was initiated, and data collection began after approval was granted by the Ethics Committee of the Dokuz Eylül University Faculty of Medicine, Non-Interventional Research Ethics Committee (approval number: 2018/09-24). Informed consent was obtained from all parents enrolled in the study.

\section{RESULTS}

\section{Demographic and Clinical Features}

Included in the study were 32 patients with classical PKU diagnosed during newborn screening. The median age at the time of diagnosis was 10 days (range: 2-16 days), and all diagnoses were made based on biochemical and genetic analyses (Phe $>1200 \mu \mathrm{mol} / \mathrm{L}$ for all). The mean age of the patients was $11.5 \pm 4.3$ years, and 14 (44\%) were female and 18 (56\%) were male. All of the patients had been on a strict low-Phe diet since the newborn period (10 g/day of natural protein), and all were receiving Phefree L-amino acid supplements in 3-4 servings/day. The mean plasma Phe level over the previous year was $732.4 \pm 339.0 \mu \mathrm{mol} / \mathrm{L}$ in all patients. Demographic characteristics of the 32 caregivers are presented in Table 1.

\section{Family Dietary Patterns}

The meals were prepared together by the mother and father in one family, while the mother was the only person preparing the meals in all other families. Considering the eating habits of the families, only low-protein meals were prepared at home for the 2 patients with PKU who had no siblings and who were living only with their parents, and all members of these 2 (6\%) families were undergoing Phe-restricted diet therapy. The mean Phe level over the last year was $351.1 \pm 68.3 \mu \mathrm{mol} / \mathrm{L}$ and $308.7 \pm 52.2 \mu \mathrm{mol} / \mathrm{L}$ in the children with PKU of these 2 families, and the Phe level of these 2 patients with PKU never exceeded $600 \mu \mathrm{mol} / \mathrm{L}$. In 26 (81\%) families, the parents prepared separate meals for the patient with PKU and for the other family members every day, and these patients had a mean Phe level of $744.5 \pm 332.9 \mu \mathrm{mol} / \mathrm{L}$. Four (12\%) parents were not preparing Phe-restricted diet meals at home. Low-Phe foods were given to the patient with PKU from the same table, while protein-rich foods were shared among other family members. These patients had a mean Phe level of $859.6 \pm 520.6$ $\mu \mathrm{mol} / \mathrm{L}$. When the 3 eating habits were compared, no statistically significant difference could be established in the respective Phe levels ( $p=0.086)$. Of the total, $3(9 \%)$ patients with PKU did not eat their meals with their families, and did not sit at the same table, 
and the mean Phe level of these 3 respondents was $889.8 \pm 351.1$ $\mu \mathrm{mol} / \mathrm{L}$. The children with PKU of 29 (91\%) parents who shared the same table with other family members had a mean Phe level of $720.3 \pm 284.5 \mu \mathrm{mol} / \mathrm{L}(\mathrm{p}=0.407)$.

When the content of the foods included in the meals of families with a patient with PKU was examined, 20 (62\%) families consumed meat once a week or less, and the mean Phe level was $768.8 \pm 302.6 \mu \mathrm{mol} / \mathrm{L}$ in the children with PKU of this group, compared to $678.0 \pm 411.6 \mu \mathrm{mol} / \mathrm{L}$ in the children with PKU of families who consumed meat more often than once a week. An examination of vegetable consumption in the home revealed that 19 (59\%) families ate vegetables every day, and 8 (25\%) families every other day. The mean Phe levels of the children with PKU in

\begin{tabular}{|c|c|}
\hline & Caregivers \\
\hline & n (\%) \\
\hline \multicolumn{2}{|l|}{ Sex } \\
\hline Female & $20(62)$ \\
\hline Male & $12(37)$ \\
\hline \multicolumn{2}{|l|}{ Age (years) } \\
\hline Mean \pm SD & $40.0 \pm 8.2$ \\
\hline $20-29$ & $2(6)$ \\
\hline 30-39 & $18(56)$ \\
\hline $40-49$ & $6(19)$ \\
\hline $50-59$ & $6(19)$ \\
\hline \multicolumn{2}{|l|}{ Domicile } \\
\hline Town & $18(56)$ \\
\hline Village & $9(28)$ \\
\hline Rural & $5(15)$ \\
\hline \multicolumn{2}{|l|}{ Educational } \\
\hline Elementary/occupational school & $26(81)$ \\
\hline Upper secondary school & $4(12)$ \\
\hline University & $2(6)$ \\
\hline \multicolumn{2}{|l|}{ Number of children } \\
\hline 1 & $3(9)$ \\
\hline 2 & $15(47)$ \\
\hline 3 & $10(31)$ \\
\hline$\geq 4$ & $4(13)$ \\
\hline \multicolumn{2}{|l|}{ Household, n (\%) } \\
\hline$\leq 4$ & $17(53)$ \\
\hline$>4$ & $15(47)$ \\
\hline \multicolumn{2}{|l|}{ Employment status } \\
\hline Full-time or part-time work & $7(22)$ \\
\hline Unemployed & $25(78)$ \\
\hline \multicolumn{2}{|l|}{ SD: Standard deviation } \\
\hline
\end{tabular}

these families were $714.3 \pm 357.1 \mu \mathrm{mol} / \mathrm{L}$ and $835.4 \pm 332.9 \mu \mathrm{mol} / \mathrm{L}$, respectively. Vegetables were consumed less frequently in 5 (16\%) families, and the mean Phe level of the children with PKU in these families was $743.5 \pm 387.4 \mu \mathrm{mol} / \mathrm{L}$.

\section{Parental Dietary Regimen}

When the parental dietary regimens were examined, 3 (9\%) families described themselves as vegan and $1(3 \%)$ as pescatarian. The children with PKU in these families had a mean Phe level of $429.8 \pm 115.0 \mu \mathrm{mol} / \mathrm{L}$ over the previous year. Among the total parent sample, 28 (88\%) had no special dietary regimen, and the children with PKU in these families had a mean Phe level of $774.8 \pm 345.4 \mu \mathrm{mol} / \mathrm{L}$. The difference between the two groups was statistically insignificant ( $p=0.055)$.

Twenty-six (81\%) were careful about the foods they consumed while eating with the patient with PKU, opting especially for foods that were low in Phe. The mean Phe level over the preceding year was $762.7 \pm 351.1 \mu \mathrm{mol} / \mathrm{L}$ in the children with PKU of the parents who were careful about the foods they consumed while eating with the patient with PKU, compared to $599.3 \pm 308.7 \mu \mathrm{mol} / \mathrm{L}$ in the children of the parents who paid no such attention $(p=0.392)$.

\section{Food Allergies}

None of the parents had a history of food allergy.

\section{DISCUSSION}

The parents of patients with PKU pay attention to the foods they consume when eating in the presence of their children. Some parents prepare only Phe-restricted diet meals at home, meaning that all family members follow a protein-restricted diet. The mean Phe level is at the target value of $<360 \mu \mathrm{mol} / \mathrm{L}$ specified in the PKU guidelines in patients with PKU of these parents. The Phe levels were lower in these families than in the other families, but statistical significance could not be obtained due to the small sample size. Some families opted not to share the same table with the patient with PKU and eat their meals at separate times or in a different room.

It has long been known that living with a child with a chronic condition imposes a psychosocial burden on families, ${ }^{4,5}$ and that lifelong diet therapy affects the quality of life of both the patient and the family. ${ }^{6}$ Parents believe that the preservation of neurocognitive functions in their children with PKU depends on successful dietary therapy ${ }^{7}$ and so families may change their lifestyles to fully comply with the diet therapy. After diagnosis, parents may change their employment attributes, preferring to work shorter hours, or quitting their job completely ${ }^{8}$ and social isolation is common in the families of a person with PKU. ${ }^{9}$ Most of the parents in the study opted to prepare separate meals for their children with PKU every day. Parents of patients with PKU spend an average of 3 hours a day preparing meals ${ }^{8}$ and struggle to find time for themselves. In the present study, the person who prepared the meals in the families of patients with PKU was 
usually the mother. Mothers work harder than fathers, and the daily lives of mothers of patients with classical PKU is affected to a greater degree than the fathers, and mothers also experience more anxiety and depression. ${ }^{10}$

Different diets evoke different feelings in patients with PKU. Especially during the adolescence, $30 \%$ of patients feel shame at not being able to consume all types of food, and their parents experience similar feelings. ${ }^{11}$ Parents who worry that their children may feel different because of the PKU diet are in a constant struggle. ${ }^{7}$ When preparing meals for their children with PKU, they try to make meals that are similar to what the other members of the family are eating. They may also modify their lifestyles such as by not consuming foods that are rich in Phe and not going to restaurants. ${ }^{7,9}$ In addition to these strategies, it was reveled in the present study that most parents imitated their children with PKU in their food preferences.

When the Phe values were examined according to the frequency of consumption of meat and vegetables in the meals of the families, no statistical difference was found. Phe level in the patients with PKU of families who consumed vegetables every day was detected slightly less than patients with PKU in families who consumed vegetables every other day or less frequently. However, Phe values were unexpectedly slightly lower in families that consumed meat more frequently than in other families. Many factors including small sample size in this study, socio-economic levels of families, diet adherence of patients with PKU, etc might cause this contradictory result.

It was a well-known fact that the food preferences of parents influenced those of their children, ${ }^{12,13}$ although in the present study it was noted that the parents adjusted their food preferences to suit those of the child with PKU in the family. Although the food preferences and food neophobias in the families of patients with PKU were found to have only a limited effect on the children's eating habits, ${ }^{14}$ the Phe levels were under control in the 2 families who followed a Phe-restricted diet as a family. When the parents opted a Phe-restricted diet, such as pescatarian, vegan or vegetarian regimens, their children with PKU had lower Phe levels than other individuals.

\section{Study Limitations}

There were certain limitations in our study, the most significant of which was the small sample size. Another limitation was that the eating habits of the sample were not compared with those with family members with healthy children. A study investigating the eating habits of all family members may be helpful in making a clear assessment of the different eating habits within the family.

\section{CONCLUSION}

Living with a patient with classical PKU places a considerable burden on the parents, affecting many different areas. While diet is a vital element in PKU treatment, it may also affect the dietary patterns of the child's parents. Large-scale studies are needed to identify the physical, social and psychological effects of parents' dietary habits on patients with classical PKU.

\section{Ethics}

Ethics Committee Approval: Ethic approval was granted by the Ethics Committee of the Dokuz Eylül University Faculty of Medicine, Non-Interventional Research Ethics Committee (approval number: 2018/09-24).

Informed Consent: Informed consent was obtained from all parents enrolled in the study.

Peer-reviewed: Externally and internally peer-reviewed.

\section{Authorship Contributions}

Surgical and Medical Practices: P.T.K., N.A., Concept: P.T.K., N.A., Design: P.T.K., N.A., Data Collection or Processing: P.T.K., A.Ç., H.K., M.D., A.G., G.Y., Analysis or Interpretation: P.T.K., N.A., Literature Search: P.T.K., N.A., Writing: P.T.K., N.A.

Conflict of Interest: The authors declared no potential conflicts of interest with respect to the research, authorship, and/or publication of this article.

Funding: The authors received no financial support for the research, authorship, and/or publication of this article.

\section{REFERENCES}

1. van Wegberg AMJ, MacDonald A, Ahring K, et al. The complete European guidelines on phenylketonuria: diagnosis and treatment. Orphanet J Rare Dis. 2017;12:162.

2. de Groot MJ, Hoeksma M, Blau N, Reijngoud DJ, van Spronsen FJ. Pathogenesis of cognitive dysfunction in phenylketonuria: Review of hypotheses. Mol Genet Metab. 2010;99:S86-S89.

3. MacDonald A, Van Wegberg AMJ, Ahring K, et al. PKU dietary handbook to accompany PKU guidelines. Orphanet J Rare Dis. 2020;15:171.

4. Wallander JL, Varni JW. Effects of pediatric chronic physical disorders on child and family adjustment. J Child Psychol Psychiatry. 1998;39:29-46.

5. Raina P, O'Donnell M, Rosenbaum P, et al. The health and well-being of caregivers of children with cerebral palsy. Pediatrics. 2005;115:e626 LPe636.

6. Fabre A, Baumstarck K, Cano A, et al. Assessment of quality of life of the children and parents affected by inborn errors of metabolism with restricted diet: preliminary results of a cross-sectional study. Health Qual Life Outcomes. 2013;11:158.

7. Carpenter K, Wittkowski A, Hare DJ, et al. Parenting a Child with Phenylketonuria (PKU): an Interpretative Phenomenological Analysis (IPA) of the Experience of Parents. J Genet Couns. 2018;27:1074-86.

8. MacDonald A, Smith TA, de Silva S, Alam V, van Loon JMT. The personal burden for caregivers of children with phenylketonuria: A cross-sectional study investigating time burden and costs in the UK. Mol Genet Metab Reports. 2016;9:1-5.

9. Ford S, O'Driscoll M, MacDonald A. Living with Phenylketonuria: Lessons from the PKU community. Mol Genet Metab Reports. 2018;17:57-63.

10. Gunduz M, Arslan N, Unal O, Cakar S, Kuyum P, Bulbul SF. Depression and anxiety among parents of phenylketonuria children. Neurosciences (Riyadh). 2015;20:350-6.

11. Witalis E, Mikoluc B, Motkowski R, et al. Phenylketonuria patients' and their parents' knowledge and attitudes to the daily diet - multi-centre study. Nutr Metab (Lond). 2017;14:57. 
12. Pearson N, Biddle SJH, Gorely T. Family correlates of fruit and vegetable consumption in children and adolescents: a systematic review. Public Health Nutr. 2009;12:267-83.

13. Damiano SR, Hart LM, Paxton SJ. Correlates of parental feeding practices with pre-schoolers: parental body image and eating knowledge, attitudes, and behaviours. Appetite. 2016;101:192-98.
14. Evans S, Daly A, Chahal S, Ashmore C, MacDonald J, MacDonald A. The influence of parental food preference and neophobia on children with phenylketonuria (PKU). Mol Genet Metab Rep. 2017;14:10-4. 